\title{
ArcheoSciences
}

Revue d'archéométrie

$36 \mid 2012$

Varia

\section{Caractérisation de phénomènes anthropiques par la mesure de paramètres magnétiques sur surface décapée : Premiers résultats sur le projet Canal Seine-Nord Europe}

Characterisation of anthropogenic impact on excavated areas by magnetic parameters measurement: First results on the Canal Seine-Nord Europe project

Hulin Guillaume, Broes Frédéric et Fechner Kai

\section{OpenEdition}

Journals

\section{Édition électronique}

URL : http://journals.openedition.org/archeosciences/3744

DOI : $10.4000 /$ archeosciences.3744

ISBN : 978-2-7535-2243-5

ISSN : 2104-3728

Éditeur

Presses universitaires de Rennes

Édition imprimée

Date de publication : 31 décembre 2012

Pagination : 61-70

ISBN : 978-2-7535-2241-1

ISSN : 1960-1360

Référence électronique

Hulin Guillaume, Broes Frédéric et Fechner Kai, «Caractérisation de phénomènes anthropiques par la mesure de paramètres magnétiques sur surface décapée : Premiers résultats sur le projet Canal Seine-Nord Europe », ArcheoSciences [En ligne], 36 | 2012, mis en ligne le 31 décembre 2014, consulté le 30 avril 2019. URL : http://journals.openedition.org/archeosciences/3744 ; DOI : 10.4000/ archeosciences.3744 


\title{
Caractérisation de phénomènes anthropiques
} par la mesure de paramètres magnétiques sur surface décapée : premiers résultats sur le projet Canal Seine-Nord Europe

\author{
Characterisation of Anthropogenic Impact on Excavated Areas by Magnetic \\ Parameters Measurement: First Results on the Canal Seine-Nord Europe Project
}

\author{
Guillaume Hulin*, Frédéric Broes** et Kai Fechner***
}

\begin{abstract}
Résumé : La démarche suivie sur le projet Canal Seine-Nord Europe se veut différente de l'utilisation standard qui peut être faite de la géophysique. La volonté est d'intégrer cet outil au plus près de l'étude archéologique comme peuvent l'être la pédologie ou la cartographie des phosphates par exemple. Grâce à une interaction forte avec les archéologues et géo-archéologues du projet, de nouvelles problématiques ont été définies et mises en oeuvre. La plus intéressante est certainement l'emploi de la géophysique pour l'étude des surfaces décapées. Par cette approche, il s’agit non pas de détecter les structures mais d'apporter à l'archéologue une information nouvelle, complémentaire des données de fouilles. Par la mesure d'un ou de plusieurs paramètres magnétiques, des phénomènes anthropiques peuvent être mis en évidence et apporter des éléments pour la compréhension du site. Les différents cas présentés illustrent la diversité des problématiques pouvant faire intervenir la géophysique. Celles-ci sont nombreuses et l'apport scientifique particulièrement important. Tout en gardant un regard critique, l'étude sur surface décapée offre un très fort potentiel qui doit encore être développé. La comparaison des résultats géophysiques avec la cartographie des phosphates constitue un des axes majeurs de cette démarche.

Abstract: The approach adopted on the Canal Seine-Nord Europe project is different from the standard application of geophysics. The will is to integrate this tool inside the archaeological study as it is done for pedology or phosphate analysis for example. Thanks to a great interaction with archaeologists and geo-archaeologists, new issues have been defined and employed on the field. The most interesting is certainly the use of geophysics to characterise soils and archaeological layers after clearance of topsoil. Thus, the aim is not to detect features but to bring new information completing field observations. More precisely, this characterisation is essentially based on the magnetic susceptibility measurement which is closely related to anthropogenic phenomena. Different cases presented here show several issues that can call upon geophysics. Those are numerous and the scientific contribution is particularly interesting. Comparison of geophysics results with phosphate analysis constitutes one of the most interesting axes of this approach.
\end{abstract}

Mots clé : Anthropisation, couche équivalente, phosphate, prospection magnétique, surface décapée, susceptibilité magnétique.

Keywords: Anthropisation, archaeological layer, equivalent stratum, magnetic susceptibility, magnetic survey, phosphate analysis.

\footnotetext{
* Inrap, Centre archéologique, 16 rue du Général Leclerc, 80400 Croix-Moligneaux, UMR 7619 Sisyphe, Université Pierre et Marie Curie, 4 place Jussieu, 75252 Paris. (guillaume.hulin@inrap.fr)

** Inrap, Centre archéologique, 16 rue du Général Leclerc, 80400 Croix-Moligneaux. (frederic.broes@inrap.fr)

*** Inrap, Centre archéologique, 11 rue des Champs, ZI La Pilaterie, 59650 Villeneuve-d'Ascq. (kai.fechner@inrap.fr)
} 


\section{INTRODUCTION}

Les études géophysiques sur surfaces en cours de fouille sont relativement rares, la géophysique étant le plus souvent employée comme méthode de reconnaissance archéologique en préalable à une éventuelle opération archéologique. Différents paramètres magnétiques peuvent être mesurés pour l'étude sur site. La susceptibilité magnétique s'avère l'un des plus pertinents et l'un des plus simples à mesurer sur le terrain. Jusqu’à présent, la mesure de la susceptibilité magnétique a généralement été employée dans une optique de repérage, sur sol non-décapé, de zones à plus fort potentiel archéologique à l'échelle d'un site (Cowell et al., 1975; Marshall, 2001) voire même à l'échelle régionale (Clark, 1990; Marmet, 2000).

La susceptibilité magnétique est depuis longtemps reconnue comme étant un marqueur fort de l'anthropisation des sols (Le Borgne, 1955, 1965; Aitken et al., 1958; Tite et Mullins, 1971). En effet, les phénomènes de chauffe (incendie de bâtiment, zone foyère, essartage), le taux de matière organique, les activités liées au travail du fer (présence de scories ou de battitures) ou dans une moindre mesure la compaction du sol sont autant de paramètres pouvant avoir une origine anthropique et qui ont pour point commun de modifier la teneur et la composition des oxydes et hydroxydes de fer dans le sol, modifications qui se traduiront par des changements de propriétés magnétiques (Le Borgne, 1955; Mullins, 1974; Marmet, 2000; Mathé \& Lévêque, 2003).

Ainsi, par la mesure de paramètres magnétiques sur les surfaces décapées, il est possible de fournir une caractérisation du sol dans le but de mettre en avant certains phénomènes anthropiques. Cette approche s'intègre ainsi très naturellement dans la démarche archéologique standard de la même manière que la pédologie (détermination du taux d'érosion, nature des couches archéologiques et des sols naturels...) ou la cartographie des phosphates (répartition et type de certaines accumulations et activités anthropiques [Fechner, 2011; Fechner et al.,2011] ).

La plupart des travaux sur ce sujet traitent d'études très limitées spatialement, le plus souvent à l'échelle de la structure (Marmet et al., 1999; Linford, 2004) ou au mieux d'une entité archéologique tel qu'un bâtiment (Jones et al., 2010). Les opérations archéologiques en préalable à la construction du Canal Seine-Nord Europe ${ }^{1}$ ont permis et permettent encore à l'heure actuelle d'appréhender un

1. Le Canal Seine-Nord Europe constitue un tracé d'une longueur de 106 km entre Compiègne (Oise) et Aubencheul-au-Bac (Nord) pour une surface totale de 2500 ha. nombre important d'occupations humaines sur une zone restreinte géographiquement. Cet échantillon significatif d'interventions géophysiques permet un comparatif intéressant. Par l'étude de trois cas pouvant être regroupés autour de deux thématiques distinctes que sont les bâtiments et les grandes fosses, il sera possible de voir en quoi la mesure de paramètres magnétiques sur les surfaces en cours de fouille présente un intérêt pour l'étude archéologique.

\section{Méthodes}

\section{Mesure directe de la susceptibilité magnétique}

De manière générale, la susceptibilité magnétique peut être définie comme étant la capacité d'un corps à s'aimanter sous l'action d'un champ magnétique. Plusieurs méthodes peuvent être mises en ouvre pour évaluer ce paramètre. La plus répandue consiste à émettre un champ électromagnétique primaire connu et à mesurer le champ secondaire émis par le volume de sol investigué. C'est le cas notamment des appareils monospire comme le MS2D de Bartington qui a été utilisé pour la plupart des études sur le projet Canal Seine-Nord Europe. Cet appareil permet une mesure point par point de la susceptibilité magnétique volumique (uSI, unité du Système International) pour une épaisseur de sol de l'ordre de $10 \mathrm{~cm}$ avec une décroissance rapide de la réponse sur les premiers centimètres (Benech et Marmet, 1999; Lecoanet et al., 1999). La mesure sur sol décapé permet un couplage parfait de la bobine avec le sol contrairement aux mesures sur terre végétale où la rugosité du sol peut générer un biais non négligeable dans les mesures.

Sur le terrain, la visualisation des faits archéologiques permet d'adapter la collecte de points. Cette prise de mesure sélective s'avère particulièrement importante dans le cas de la caractérisation des sols où la contribution des vestiges archéologiques, des artefacts naturels évidents (chablis) et autres perturbations anthropiques (vestiges de la Première Guerre Mondiale pour ce qui concerne les zones d'étude concernées) ne doit pas être prise en compte.

Le positionnement des données de susceptibilité s'effectue en temps réel grâce à un GPS de précision décimétrique après correction différentielle en post-traitement (Trimble GeoXH 2008).

Pour certaines études, des mesures ont également été réalisées sur échantillons dans le but de compléter les données de terrain. L'utilisation du MS2B a permis la mesure de la susceptibilité magnétique massique $\left(\mathrm{m}^{3} / \mathrm{kg}\right)$ (élimination de l'effet lié au volume) ainsi que de la dépendance en fréquence. Ce paramètre, obtenu en mesurant la susceptibi- 
lité à deux fréquences différentes $(0,465 \mathrm{kHz}$ et $4,65 \mathrm{kHz})$, nous renseigne sur la taille des grains contribuant au signal magnétique (Dearing et al., 1996). Les mesures sur échantillons ont été réalisées sur la fraction inférieure à $2 \mathrm{~mm} \mathrm{du}$ sédiment sec.

\section{Mesure du champ magnétique et calcul de la susceptibilité d'une couche équivalente}

La susceptibilité magnétique peut également être appréhendée de manière indirecte par la mesure passive du champ magnétique local. En effet, celui-ci est la somme du champ magnétique terrestre (uniforme à l'échelle de la zone d'étude), des variations diurnes et du champ généré par les aimantations des différents matériaux présents dans le sol. Cette dernière contribution est isolée du reste en ramenant à zéro la médiane de chacun des profils. Ce traitement est possible grâce à la faible longueur des profils (temps d'acquisition par profil inférieur à 20 s) et de la prise de mesure lors d'une journée calme d'un point de vue magnétique (Tabbagh, 2003).

Ensuite, par un produit de convolution appliqué aux mesures de terrain, il est théoriquement possible d'obtenir une image de la répartition de l'aimantation verticale dans le sol (exprimée en $\mathrm{A} / \mathrm{m}$ ). Pour facilité l'interprétation des valeurs obtenues, celles-ci sont exprimées en termes de variations de susceptibilité magnétique (uSI) bien que d'autres aimantations aient pu contribuées à la réponse magnétique. Il est en effet évident que la part de cette aimantation totale liée à la présence d'aimantations rémanentes est non-négligeable.

La technique utilisée ici est celle de la couche équivalente de susceptibilité décrite notamment par Desvignes et al. (1999). Dans le cas présenté ici, l'épaisseur de la couche équivalente a été fixée à $10 \mathrm{~cm}$. L'échelle de valeurs a été recalée grâce à la mesure de la susceptibilité sur un échantillon de référence prélevé en dehors des anomalies de fortes aimantations. Ce procédé permet une comparaison plus facile avec les mesures directes de susceptibilité obtenues avec le MS2D.

Sur le terrain, l'enregistrement en continu par un magnétomètre à pompage optique (ici un Geometrics G-858) permet une grande densité de points (maille de $10 \times 50 \mathrm{~cm}$ ) mais empêche totalement la discrimination des données contrairement au MS2D. En effet, par cette méthode, la totalité de la zone d'étude est couverte ce qui entraine que le signal mesuré contient également les contributions des structures archéologiques et d'autres perturbations éventuelles. La mesure du champ magnétique nécessite donc une très faible pollution magnétique sur le site pour que le passage en couche équivalente de susceptibilité soit significatif. Dans tous les cas, les anomalies magnétiques de forte amplitude (y compris celles générées par les vestiges archéologiques) sont minimisées par un traitement adapté (filtrage par la médiane sur une fenêtre glissante) afin de se concentrer sur le signal généré par le sol et de tendre vers une inversion des données la plus robuste possible.

\section{Résultats}

\section{Des bâtiments néolithiques}

Le premier résultat pertinent sur le projet Canal SeineNord Europe a été obtenu sur la plate-forme PF3 de Marquion/Sauchy-Lestrée (Pas-de-Calais) lors de la phase de diagnostic. Cette étude portait sur plusieurs zones test dont une sur surface décapée et qui présente trois bâtiments datés du Néolithique final (fig. 1). Parmi ces trois bâtiments, deux sont établis sur tranchée de fondation, le troisième est bâti sur poteaux plantés (Gaillard, 2010).

Par la mesure du champ magnétique et surtout l'inversion des données selon la technique de la couche équivalente de susceptibilité, des résultats intéressants ont été obtenus. Les cartes présentées sont de deux types. La carte de champ total correspond à la carte d'anomalies mesurées à $30 \mathrm{~cm}$ au-dessus de la surface décapée. Elle permet de voir une partie des structures archéologiques. La carte de couche équivalente de susceptibilité met en avant les variations latérales d'aimantations pouvant être à l'origine des anomalies magnétiques mesurées. Il s'agit d'une solution possible du problème inverse. A noter que cette approche requiert un rapport signal/bruit particulièrement élevé ce qui est le cas sur surface décapée ${ }^{2}$. En effet, la suppression de l'horizon superficiel permet à la fois d'éliminer une source importante de bruit magnétique et de se rapprocher des niveaux archéologiques ce qui a pour conséquence d'augmenter l'amplitude du signal magnétique.

La comparaison de la carte d'anomalies avec le plan issu du diagnostic archéologique montre de manière évidente les différences entre ces deux approches (fig. 1b. et 1c.). Il est intéressant de noter que dans le cas présent, aucun des trois bâtiments n'aurait pu être identifié par la seule utilisation de la géophysique.

Le passage en couche équivalente de susceptibilité (fig. 1d.) révèle des informations plus pertinentes en montrant de manière particulièrement évidente le bâtiment 3 .

2. Seules deux anomalies fortes sont présentes sur la fenêtre sud. Celles-ci ne sont pas d'origine archéologique (clous, éclats d'obus). Elles ont été supprimées pour le calcul de la couche équivalente. 

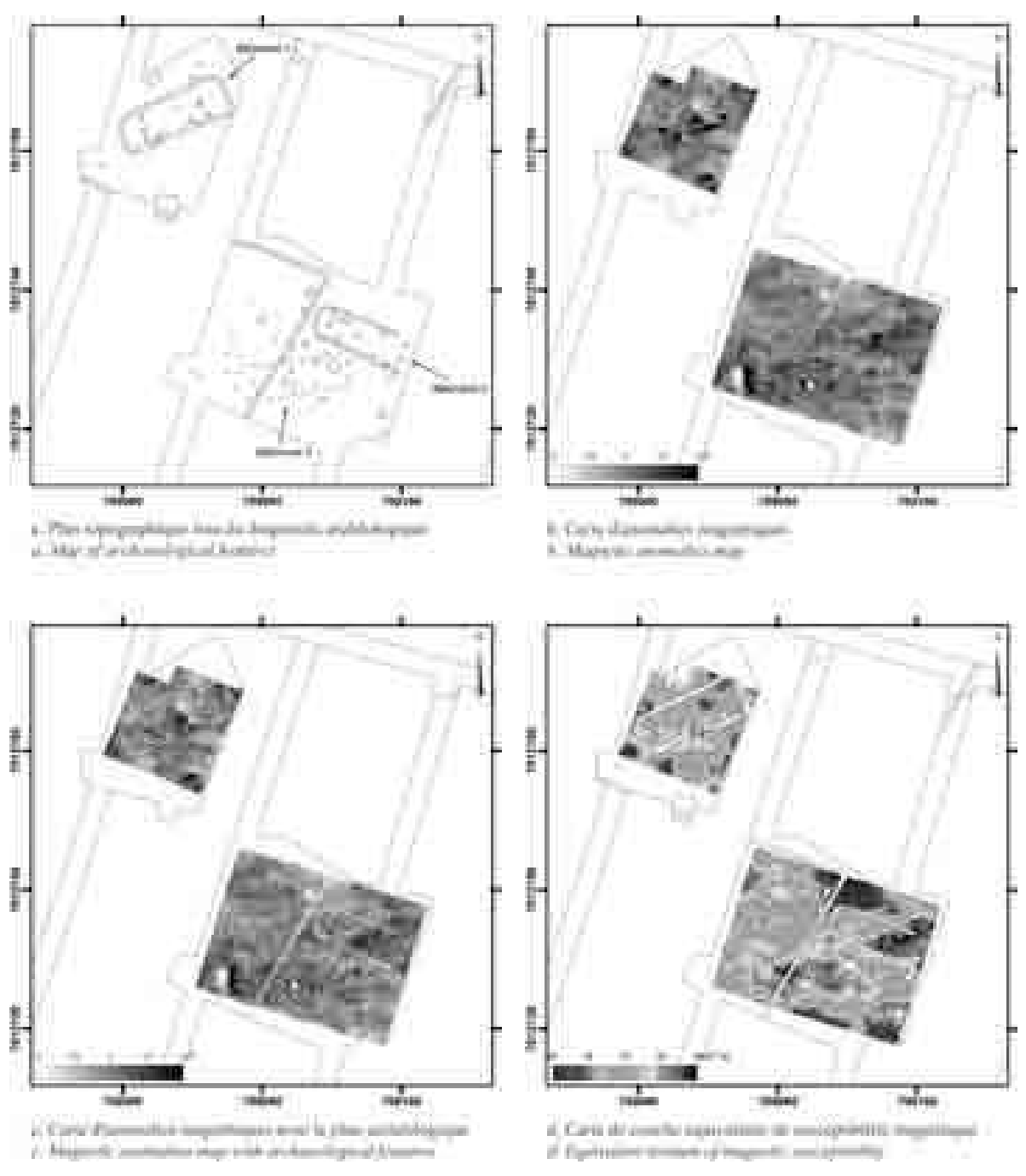

Figure 1 : (Voir planche couleur) Carte d'anomalies magnétiques et carte de la couche équivalente de susceptibilité magnétique sur des bâtiments néolithiques (PF3 Marquion/ Sauchy-Lestrée - Pas-de-Calais) Figure 1: (See colour plate) Magnetic survey and equivalent stratum of magnetic susceptibility on Neolithic buildings (PF3 Marquion/SauchyLestrée - Pas-de-Calais, France)
Ainsi, celui-ci apparaît non pas comme une succession de trous de poteau mais comme une zone de plus forte aimantation aux contours relativement bien définis.

Les deux bâtiments sur tranchée de fondation apparaissent de manière moins évidente. Cependant, on note sur le bâtiment 2 une zone de forte aimantation présentant un effet de paroi avec le côté sud du bâtiment uniquement. Ce panache pourrait être la traduction de phénomènes anthropiques bien qu'une origine naturelle ne puisse être exclue. Le bâtiment 1 présente également une zone de plus forte aimantation au niveau de l'interruption de la tranchée. La prudence est cependant de mise puisqu'il est difficile d'évaluer la part de la contribution des structures archéologiques dans l'inversion des données. En effet, celles-ci présentent un remplissage relativement magnétique.

La dynamique des valeurs obtenues pour la couche équivalente (27 à $175 \times 10-5$ uSI) est importante et ne doit pas être expliquée uniquement par la susceptibilité magnétique.
Dans le cas présent, la contribution des aimantations rémanentes et notamment de l'aimantation rémanente visqueuse est à envisager. La mesure directe de la susceptibilité magnétique lors de la phase de fouille permettra de discuter plus précisément de la part des différentes aimantations dans le signal mesuré.

Ce type de cartographie offre donc une autre perception du site archéologique au travers de paramètres physiques difficilement appréhendables autrement sur le terrain. Le cas des bâtiments néolithiques a permis de mettre en avant que la mesure de paramètres magnétiques, ici le champ magnétique local, peut apporter des informations intéressantes même après décapage de la terre végétale. Cette étude constitue le point de départ de l'ensemble de la démarche mise en œuvre sur le projet Canal Seine-Nord Europe. 


\section{Un bâtiment protohistorique}

Dans la même thématique liée à la caractérisation des bâtiments, une autre étude menée sur le projet Canal Seine-Nord Europe a montré des éléments particulièrement intéressants tant d'un point de vue archéologique que méthodologique.

Il s'agit d'un ensemble de trous de poteau observés et étudiés grâce à la fouille du site de Sauchy-Lestrée " le Grotin " (Pas-de-Calais). Ce probable bâtiment daté de l'Age du Fer est situé dans un ensemble assez lâche de vestiges traduisant une occupation humaine somme toute relativement faible (Desforges, 2011). Ce dernier point permet par ailleurs de fournir des interprétations plus fiables puisque le risque de contamination par d'autres occupations humaines se trouve minimisé. De plus, l'étude pédologique a montré que l'aire du bâtiment se trouve sur une zone où l'état de conservation est bon avec un décapage uniforme sur l'horizon Bt. La zone d'étude est centrée sur cet ensemble de poteau en formant un carré de $17 \mathrm{~m}$ de côté environ. La présence de vestiges liés à la Première Guerre mondiale a contre indiqué l'utilisation de la mesure du champ magnétique et de ce fait, l'emploi du susceptibilimètre MS2D s'est avéré le plus pertinent.

La cartographie de susceptibilité magnétique selon un maillage de l'ordre du mètre (336 points de mesure) montre des variations relatives importantes pour ce genre de contexte pédologique (fig. 2). Les données s'échelonnent sur un intervalle allant de 22 à $40 \times 10-5$ uSI avec une médiane à $31 \times 10-5$ uSI et un écart-type de $3 \times 10-5$ uSI. Une zone de plus forte susceptibilité est très clairement visible. Les valeurs $y$ atteignent 34 à $40 \times 10-5$ uSI soit une augmentation maxi- male de près de $30 \%$ par rapport à la susceptibilité magnétique moyenne. Cette anomalie présente une corrélation spatiale forte avec les deux alignements de trous de poteau observés en fouille. Des effets de paroi plutôt bien marqués sont présents au niveau de ces deux lignes de poteaux et il est particulièrement intéressant de voir que l'alignement nord se prolonge avec l'anomalie de susceptibilité et se termine en vis-à-vis du dernier poteau de l'alignement sud.

L'association de valeurs fortes et d'effets de paroi liés à des structures archéologiques conduit à interpréter ces variations comme étant le résultat d'un impact anthropique sur le sol. Comme précédemment évoqué, cet impact peut être le résultat de phénomènes divers et complexes. Dans le cas présent, il est plus vraisemblable d'associer celui-ci à une chauffe ou à la présence en plus grande quantité de matière organique (Le Borgne, 1955; Mullins, 1974; Marmet, 2000).

Afin d'affiner l'interprétation, une cartographie des phosphates a été mise en œuvre sur cette même zone. La méthode de prospection employée est basée sur un protocole proposé depuis les années 60 (Schwartz, 1968; Eidt, 1984) qui consiste en la réalisation de tests de terrain rapides et peu coûteux. Cette première phase est suivie de dosages quantitatifs du phosphate total, organique et inorganique en cas de succès des tests, c'est-à-dire de coïncidences entre des limites (effets de paroi) montrées par les tests et le plan archéologique (Devos et al., 2011; Fechner et al., 2011). Le fait de faire appel à des tests et des analyses apporte des renseignements complémentaires qui se sont avérés indispensables à cette étude. Les résultats obtenus (fig. 3) à partir de 67 échantillons montrent une hausse de la teneur en phos-
Figure 2 : (Voir planche couleur) Carte de susceptibilité magnétique sur un bâtiment protohistorique (Sauchy-Lestrée « le Grotin " - Pasde-Calais).

Figure 2: (See colour plate) Magnetic susceptibility map on a protohistoric building (Sauchy-Lestrée "le Grotin"Pas-de-Calais, France).

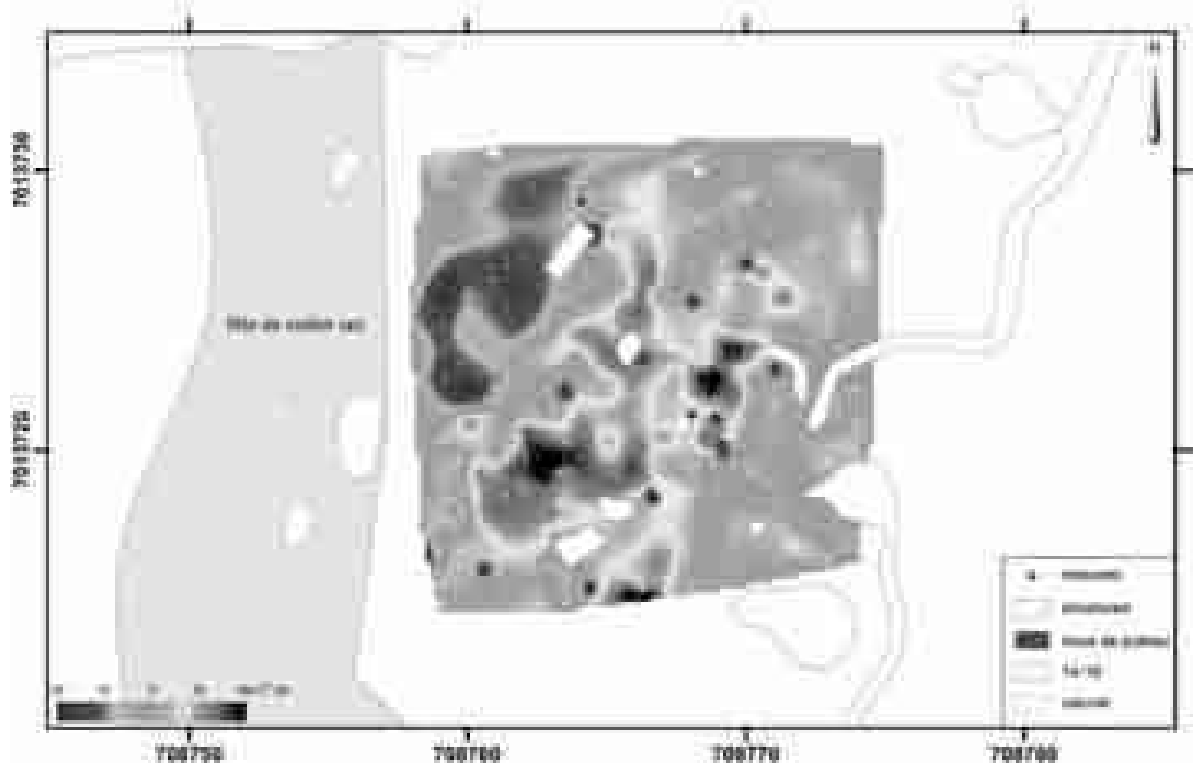

ArCheoSCIEnCes, revue d'archéométrie, 36, 2012, p. 61-70 


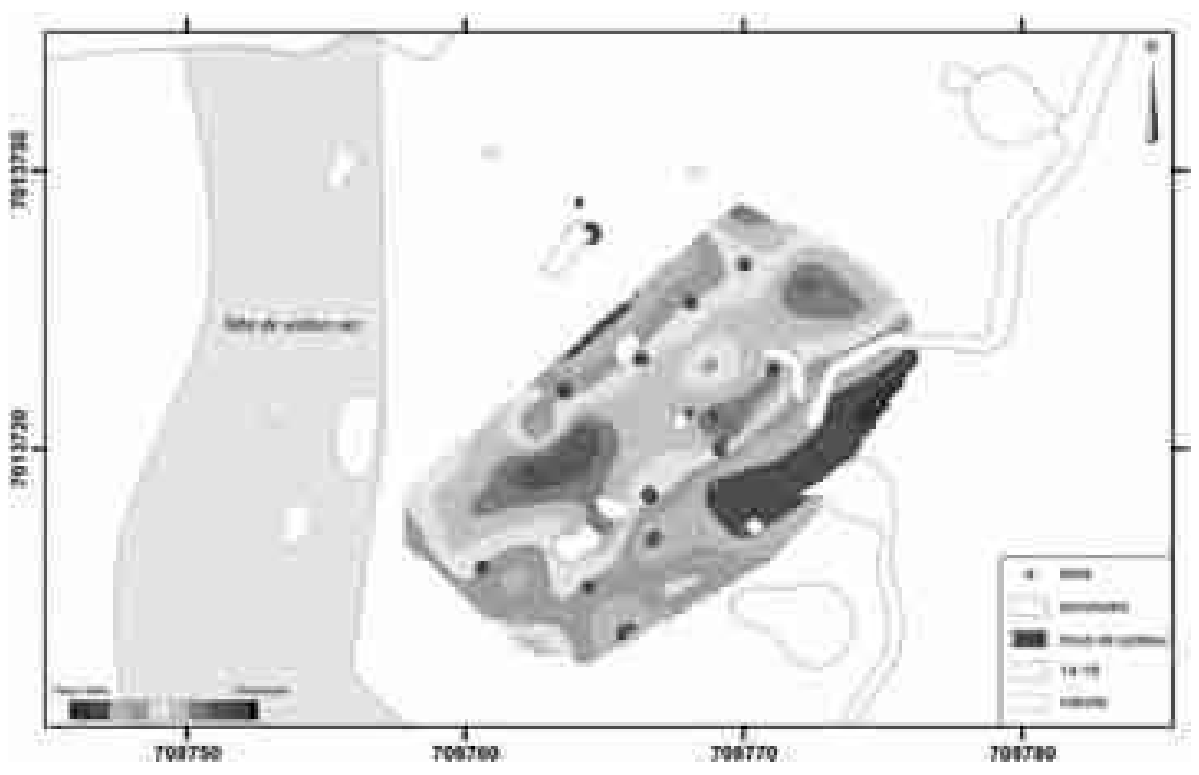

Figure 3 : (Voir planche couleur) Carte des tests de phosphore sur un bâtiment protohistorique (Sauchy-Lestrée «le Grotin »- Pas-de-Calais).

Figure 3: (See colour plate) Phosphorus tests map on a protohistoric building (Sauchy-Lestrée "le Grotin" - Pas-deCalais, France). phate ainsi que des effets de paroi qui corrèlent particulièrement bien avec les données de susceptibilité magnétique. L'approche combinée permet ainsi de mettre en évidence une accumulation ancienne d'origine anthropique préférentiellement liée à de fortes concentrations de matière organique (voir cas du Néolithique ancien comparables : Fechner et al.,2011) .

Cette étude se révèle donc particulièrement positive sur plusieurs aspects. Pour l'archéologue, elle permet de confirmer l'orientation du bâtiment repéré en fouille et même de le prolonger vers le sud-ouest ce qui n'apparaît pas évident à la seule lecture du plan de fouille. De plus, des hypothèses sur la fonction de ce bâtiment peuvent également être proposées ce qui constitue un apport majeur.

Ces résultats vont dans le même sens que ceux obtenus lors de l'étude des bâtiments néolithiques en montrant à nouveau qu'un impact relativement fort peut être mesuré sur la surface décapée. La pertinence des analyses de susceptibilité magnétique et de phosphate comme marqueurs de l'activité anthropique est prouvée depuis longtemps mais ces deux méthodes, souvent utilisées séparément sont d'un plus grand bénéfice lorsqu'elles sont combinées. Cette étude en apporte une nouvelle confirmation. In fine, l'approche combinée mérite d'être appliquée à un plus grand nombre de cas avec pour objectif la distinction entre les bâtiments ou parties de bâtiments à vocation domestique ou agricole.

\section{Des grandes fosses gallo-romaines}

La seconde problématique évoquée ici concerne l'étude de structures archéologiques dans l'optique d'apporter des éléments de compréhension par l'intermédiaire d'une caractérisation magnétique de leur remplissage. Le cas des grandes fosses ou dépressions généralement retrouvées sur les occupations gallo-romaines rentre parfaitement dans ce cadre. En effet, bien que très communément rencontrées, la ou les fonctions de ces structures archéologiques restent assez mal définies (mares, fosses d'extraction...). Le cas présenté ici est celui du site de Saint Christ-Briost "Les Dix-huit " (Somme) où une succession d'occupations matérialisées par des enclos fossoyés s'est échelonnée de la fin du i ${ }^{\text {er }}$ siècle avant notre ère jusqu'au IV $\mathrm{V}^{\mathrm{e}}$ siècle après J.-C. A l'intérieur de ces enclos, quatre grandes fosses ont pu être observées.

Létude a consisté en la réalisation d'une cartographie systématique de la susceptibilité magnétique avec une maille de mesure de l'ordre du mètre sur chacune de ces fosses (1588 points de mesure). Les mesures ont été réalisées à l'aide du susceptibilimètre MS2D (fig. 4). On constate pour trois d'entre elles un remplissage assez homogène avec des valeurs oscillant autour de 50×10-5 uSI. Une de ces fosses (St. 233) sort totalement de ce lot en présentant trois voire quatre zones internes offrant des valeurs comprises entre 100 et $200 \times 10-5$ uSI. Il faut noter que ces fortes valeurs se retrouvent sur l'intégralité de l'extension verticale de la fosse (mesures réalisées sur les coupes). Par ailleurs, cette gamme de valeurs est typique des fours domestiques présents sur le site.

Cette différence extrêmement importante possède très vraisemblablement une origine archéologique. La compréhension du phénomène en cause peut donc se révéler importante pour l'interprétation de cette structure. L'observation visuelle du sédiment a montré qu'à l'emplacement des zones 
Figure 4: (Voir planche couleur) Carte de susceptibilité magnétique sur les grandes fosses superposée à la photographie aérienne et au plan archéologique (Saint ChristBriost « les Dix-huit "-Somme).

Figure 4: (See colour plate) Magnetic susceptibility map on large pits overlayed with aerial photography and archaeological map (Saint Christ-Briost "les Dix-huit"-Somme, France).

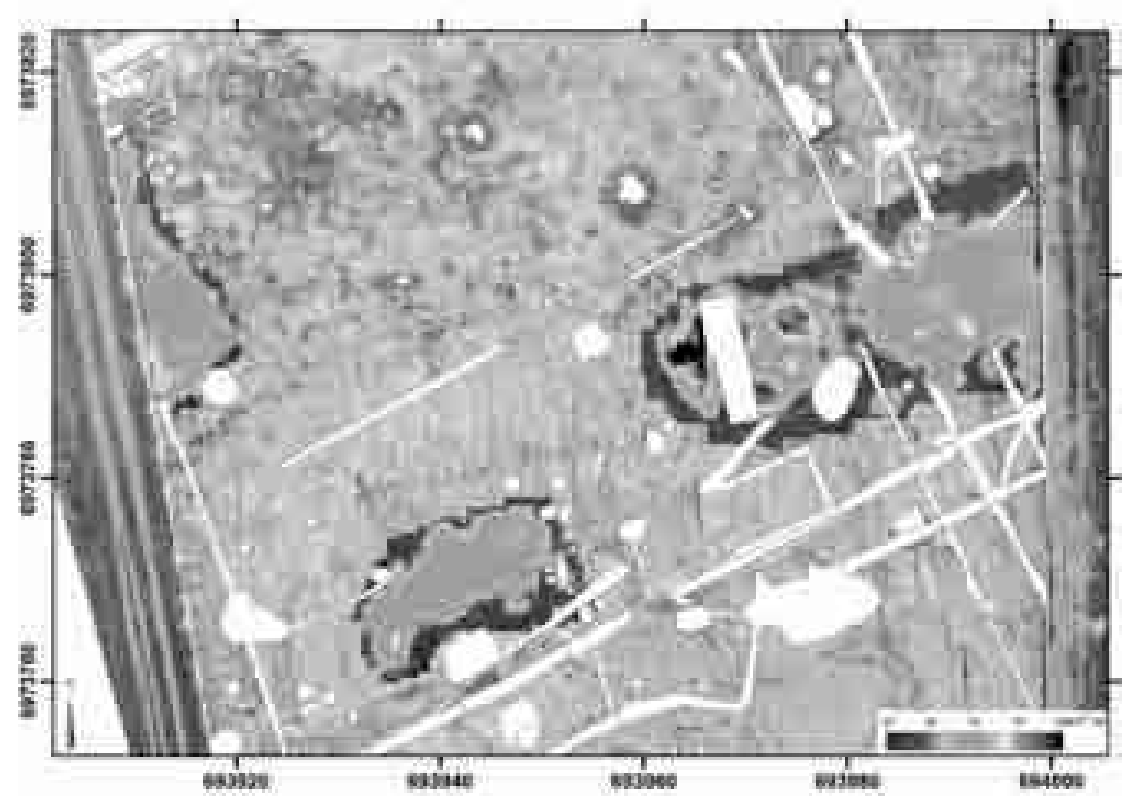

magnétiquement plus fortes des graines carbonisées étaient présentes en grande quantité. Relier la concentration de graines carbonisées à une plus forte aimantation du remplissage sédimentaire est une piste de travail intéressante qu'il convient d'approfondir. Il est notamment reconnu que la présence de matière organique joue un rôle important dans l'acquisition de l'aimantation lors de la chauffe d'un sédiment en modifiant les conditions d'oxydo-réduction (Le Borgne, 1960; Mullins, 1974; Marmet, 2000; Weston, 2002). Ainsi, la réduction de l'hématite en magnétite qui a lieu aux alentours de $500^{\circ} \mathrm{C}$ est très nettement favorisée par la présence de matière organique.

Une série de prélèvements a été réalisée afin de compléter les données de terrain par des mesures au MS2B ainsi que des mesures des taux de carbone organique et d'azote (mesures chimiques réalisées à l'INRA d'Arras). Ceci dans le but d'évaluer une éventuelle corrélation entre matière organique et susceptibilité magnétique (tab. 1).

Les mesures de la susceptibilité magnétique sur échantillons suivent parfaitement les mesures de terrain avec cependant quelques variations dues aux différences de volumes pris en compte par les deux capteurs. Les valeurs de dépendance en fréquence ne présentent aucune variation significative ce qui limite l'intérêt de l'étude de ce paramètre.

Les analyses chimiques montrent des résultats intéressants. La susceptibilité magnétique massique et le taux de matière organique présentent un coefficient de corrélation de 0,48 ce qui est significatif vu le nombre d'échantillons (à un niveau de confiance de $90 \%$ ) mais d'autres informations seraient nécessaires pour expliquer la dispersion. Le rapport
Carbone/Azote montre quant à lui un coefficient de corrélation de 0,71 avec la susceptibilité magnétique massique. Cela suggère que les fortes valeurs de susceptibilité magnétique seraient ici liées à une moins bonne décomposition de la matière organique.

Il semble donc plus pertinent d'associer les fortes valeurs de susceptibilité magnétique à une chauffe du sédiment. Les analyses chimiques ont montré que la teneur en matière organique était partiellement corrélée aux variations de susceptibilité. Sans être la cause principale, la présence de matière organique dans le sédiment pourrait avoir facilité l'augmentation du signal magnétique lors de la chauffe de celui-ci (Le Borgne, 1960; Mullins, 1974; Marmet, 2000).

Excepté la présence de graines carbonisées, il est important de signaler que les observations de terrain n'ont pas permis de déceler de traces de chauffe que ce soit en surface ou en coupe. Cependant, la cohérence et surtout les très fortes valeurs mesurées permettent d'affirmer qu'il s'agit bien de la signature d'un acte anthropique. Par ailleurs, les mesures magnétiques ne permettent pas de déterminer si le ou les phénomènes responsables de cette forte hausse de susceptibilité magnétique ont eu lieu sur place ou non.

\section{Discussion}

Les trois exemples présentés ici montrent l'apport que peut avoir la mesure de paramètres magnétiques sur les surfaces en cours de fouille. Par certains aspects, l'étude sur les grandes fosses rejoint des précédents travaux qui visaient 


\begin{tabular}{|c|c|c|c|c|c|c|c|}
\hline Prélèvement & $\begin{array}{c}\text { Susceptibilité } \\
\text { volumique } \\
\text { terrain) } \\
\times 10-5 \mathrm{uSI}\end{array}$ & $\begin{array}{c}\text { Susceptibilité } \\
\text { massique } \\
\times 10-8 \mathrm{~m} 3 / \mathrm{kg}\end{array}$ & $\begin{array}{c}\text { Dépendance } \\
\text { fréquentielle } \%\end{array}$ & $\begin{array}{c}\text { Carboneor ga- } \\
\text { nique } \\
\mathrm{g} / \mathrm{kg}\end{array}$ & $\begin{array}{c}\text { Azotet otal } \\
\mathrm{g} / \mathrm{kg}\end{array}$ & $\begin{array}{c}\text { Matièreor ganique } \\
\mathrm{g} / \mathrm{kg}\end{array}$ \\
\hline P1 & 122 & 119 & 9.5 & 8.63 & 0.696 & 12.4 & 14.9 \\
\hline P2 & 86 & 72.3 & 8.02 & 6.24 & 0.634 & 9.84 & 10.8 \\
\hline P3 & 144 & 178.8 & 8.28 & 11.1 & 0.986 & 11.3 & 19.2 \\
\hline P4 & 54 & 31.2 & 8.33 & 10.2 & 1.13 & 9.04 & 17.7 \\
\hline P5 & 70 & 54.7 & 8.59 & 7.84 & 0.802 & 9.78 & 13.6 \\
\hline P6 & 80 & 67.3 & 8.92 & 8.2 & 0.825 & 9.94 & 14.2 \\
\hline P7 & 106 & 95.1 & 9.04 & 7.52 & 0.759 & 9.91 & 13 \\
\hline P8 & 48 & 41.3 & 8.23 & 6.62 & 0.746 & 8.88 & 11.5 \\
\hline P9 & 100 & 99 & 9.09 & 9.21 & 0.885 & 10.4 & 15.9 \\
\hline P10 & 45 & 41.9 & 7.64 & 5.41 & 0.61 & 8.86 & 9.36 \\
\hline P11 & 68 & 63.2 & 8.54 & 6.73 & 0.625 & 10.8 & 11.6 \\
\hline P12 & 97 & 78.3 & 8.68 & 10.5 & 0.931 & 11.2 & 18.1 \\
\hline P13 & 76 & 71.8 & 9.33 & 8.89 & 0.897 & 9.91 & 13.4 \\
\hline P14 & 77 & 59.8 & 8.53 & 7.93 & 0.797 & 9.96 & 13.7 \\
\hline P15 & 68 & 74.8 & 8.42 & 5.77 & 0.637 & 9.07 & 9.99 \\
\hline
\end{tabular}

Tableau 1 : Mesures physico-chimiques sur échantillons issus de la fosse 233 (Saint Christ-Briost « Les Dix-huit » - Somme) Tableau 1: Physico-chemical measurements of samples from the pit 233 (Saint Christ-Briost "Les Dix-huit" - Somme, France)

à caractériser certaines structures archéologiques (Marmet, 1999).

L'étude sur les bâtiments constitue un aspect plus original en montrant qu'un impact relativement fort peut être mesuré en dehors de tout témoin archéologique observable. Les apports scientifiques peuvent s'avérer particulièrement nombreux et ce d'autant plus que la surface étudiée sera large. Ainsi, la mesure de paramètres magnétiques pourrait, par exemple, permettre de voir des différences inter et/ou intra bâtiments ou bien encore aider à délimiter des aires au sein d'ensembles homogènes d'entités archéologiques.

Bien que le lien avec l'entité archéologique semble évident, l'interprétation des données peut être rendue délicate par la complexité des phénomènes qui entrent en jeu dans le cycle d'oxydation-réduction des grains d'oxydes de fer. Ainsi, une variation de susceptibilité magnétique ne pourra être interprétée comme étant d'origine archéologique que si au moins une des conditions suivantes est présente :

- Corrélation spatiale des données magnétiques avec des structures archéologiques (effets de paroi).

- Valeurs anormalement élevées ne pouvant être dues qu’à des phénomènes anthropiques (chauffe, présence de battitures)

Le principal argument en faveur d'une datation ancienne des variations de susceptibilité magnétique tout comme des enrichissements en phosphore est la bonne corrélation avec des limites archéologiques (de fosse, d'unité architecturale, d'enclos) et l'absence de corrélation avec des limites plus récentes (de parcelle, de chemin...). On exclut par la même occasion la possibilité d'un phénomène lié à un marquage antérieur ou postérieur aux occupations anciennes. Il est évident que par ces méthodes, on étudie le bilan de ces occupations sans toujours pouvoir identifier une éventuelle succession de différentes phases.

Il semble ainsi plus judicieux de ne réaliser une étude de susceptibilité qu’à partir d'entités archéologiques clairement définies par la fouille. La démarche inverse, consistant à se baser sur les résultats issus de l'étude géophysique pour rechercher d'éventuels indices d'entités archéologiques semble, à l'heure actuelle, trop hasardeuse.

Les résultats des études menées sur les sites du Canal Seine-Nord Europe montrent que les deux approches, susceptibilité magnétique et analyse du phosphore, ont les mêmes facteurs de réussite. Lorsque le site est implanté sur un sol encaissant unique (ici des limons décarbonatés sur une profondeur de 40 à $140 \mathrm{~cm}$ ) et en contexte de plateau, de sommet de versant ou de fond de vallée plat et sec, l'absence d'érosion supérieure à $40 \mathrm{~cm}$ permet de conserver des traces d'occupations humaines et ce même en dehors des faits archéologiques observés. De plus, sur ce type de sol à bonne fertilité chimique et physique, le mélange séculaire des premiers décimètres de sol par la bioturbation a permis 
une conservation des traces physico-chimiques à cartographier sur une plus grande profondeur.

Il est important d'insister sur le fait que la compréhension des variations des paramètres magnétiques et la fiabilité des interprétations ne pourront se faire que par la multiplication des études. Les travaux entrepris sur le projet Canal SeineNord Europe y contribuent. Dans les deux cas présentés sur les bâtiments, ce sont deux méthodes différentes qui ont été utilisées ce qui ne permet pas une véritable comparaison. Il convient donc d'asseoir la démarche par des études comparatives strictes. Il semble primordial de coupler les mesures d'anomalies magnétiques avec les mesures directes de susceptibilité lorsque les contingences techniques le permettent afin de déterminer ce que leur association pourrait apporter. Ce couplage méthodologique pourrait notamment offrir la possibilité de faire la distinction entre aimantation induite d'une part et aimantations rémanentes d'autre part (Pétronille et al., 2010). Pour exemple, il a pu être prouvé que l'aimantation rémanente visqueuse peut atteindre une amplitude équivalente à celle de l'aimantation induite sur une période de 1000 à 2000 ans (Mullins, 1974; Thiesson, 2007). La profondeur d'investigation, différente pour les deux méthodes, devra également être prise en compte pour l'interprétation de ces résultats couplés. Enfin, la réalisation de cartographies des phosphates constitue un second axe majeur de cette démarche qu'il convient de développer pour aider à mieux caractériser les anomalies observées. A ce jour, encore trop peu d'expériences de ce type ont été réalisées (Smith et al., 2001, Jones et al., 2010, Fechner et al., 2011).

\section{Conclusion}

Par l'étude de trois cas issus des travaux menés sur le projet Canal Seine-Nord Europe, il a été possible de constater qu'un impact anthropique relativement fort peut être mis en évidence sur surface décapée grâce à la mesure du signal magnétique. Un tel apport d'informations semble particulièrement intéressant en offrant une vision complémentaire pour la compréhension du site. Les problématiques pouvant être abordées par ces mesures sont particulièrement nombreuses et ne s'arrêtent pas qu'aux deux thématiques présentées ici. Les approches liées au travail du fer en constitue l'une des plus évidentes.

\section{Remerciements}

Nos remerciements s'adressent particulièrement à Alain Tabbagh et Julien Thiesson pour leurs conseils et leur relecture attentive de cet article.

\section{Bibliographie}

Aitken, M. J., Webster, G. et Reeds, A., 1958 - Magnetic prospecting, Antiquity, 32, p. 270-271.

Benech, C. et Marmet, E., 1999 - Optimum depth of investigation and conductivity response rejection of the different electromagnetic devices measuring apparent magnetic susceptibility, Archaeological Prospection, 6, p. 31-45.

Clark, A., 1990 - Seeing beneath the soil: prospecting methods in archaeology, Batsford, London.

Cowell, M. R., Craddock, P. T., Hughes, M. J., Sieveking, G. G. et Tite, M.S., 1975 - Soil phosphate analysis as a survey technique and comparison with magnetic susceptibility data, In 15th Symposium on archaeometry and archaeological prospection, $\mathrm{O}$ xford.

Dearing, J.A., Dann, R. J. L., Hay, K., Lees, J. A., Loveland, P. J., Mahe, B. A. et O'Grady, K., 1996 - Frequency-dependent susceptibility measurements of environmental materials, Geophysical Journal International, 124, p. 228-240.

Desvignes, G., Tabbagh, A. et Benech, C., 1999 - The determination of the depth of magnetic anomaly sources, Archaeological Prospection 6, p. 86-105.

Desforges, J.-D., 2011 - Les marges d'une ferme indigène à Sauchy-Lestrée, Canal Seine-Nord Europe, fouille 5, Inrap, Service Régional de l'Archéologie.

Devos, Y., Fechner, K. et Miknelsen, J. H., 2011 - The application of phosphorus cartography to archaeological structures: the development of a protocol, dans Fechner, K., Devos, Y., Leopold, M., Völkel, J. (ed.). Archaeology, soil- and lifesciences applied to enclosures and fields. Proceedings of the Session 'From microprobe to spatial analysis - Enclosed and buried surfaces as key sources in Archaeology and Pedology', European Association of Archaeologists, $12^{\text {th }}$ Annual Meeting, Krakow, 2006. BAR International Series, 2222, p. 9-28.

EIDT, R.C., 1984 - Advances in abandoned settlement analysis. Application to Prehistoric Anthrosols in Colombia, South America, Chapter 4: methodology of phosphate analysis: field application. University of Wisconsin-Milwaukee.

FeChNer, K., 2011 - Case studies of phosphorus mapping in Late La Tène to Roman enclosures and ponds between Rhine and Seine, dans Fechner, K., Devos, Y., Leopold, M., Völkel, J. (ed.). Archaeology, soil- and life-sciences applied to enclosures and fields. Proceedings of the Session 'From microprobe to spatial ana- 
lysis - Enclosed and buried surfaces as key sources in Archaeology and Pedology', European Association of Archaeologists, 12th Annual Meeting, Krakow, 2006. BAR International Series, 2222, p. 29-64.

Fechner, K., de Lil, A., Clavel, V., Hus, J. et Teheux, E., (avec les collaborations de F. Broes, F. Dugois (†), E. Elleboode, S. Fournand, D. Gaillard, F. Heller, M. Julien, E. LeroyLangelin, Y. Lorin, J.-Ph. Marchal, H.-G. Naton, Ch. Poirier, I. Praud, J.-L. Slachmuylder) 2011 - Méthodes et résultats de la cartographie du phosphore dans des bâtiments allongés du Néolithique dans le nord de la France et en Wallonie, dans Bostyn, F., Martial, E., Praud, I. (dir.). Le Néolithique du nord de la France dans son contexte européen: habitat et économie aux $4 \mathrm{e}$ et 3e millénaires avant. Actes du $29^{\mathrm{e}}$ Colloque interrégional sur le Néolithique, Villeneuved'Ascq, 2-3 octobre 2009. Revue Archéologique de Picardie, 28, p. 275-298.

GAILlaRD, D., 2010 - Recherches sur les occupations humaines du Néolithique au Haut Moyen Age sur les terroirs de Marquion et de Sauchy-Lestrée, Canal Seine-Nord Europe, PF3, Marquion Sauchy-Lestrée (62), Tome II-1, Inrap, Service Régional de l'Archéologie.

Jones, R., Challands, A., French, C., Card, N., Downes, J., Richards, C., 2010 - Exploring the location and function of a Late Neolithic house at Crossiecrown, Orkney by geophysical, geochemical and soil micromorphological methods, Archaeological Prospection, 17, p. 29-47.

Le Borgne, E., 1955 - Susceptibilité magnétique anormale du sol superficiel, Annales de Géophysique, 11, p. 399-419.

Le Borgne, E., 1960 - Influence du feu sur les propriétés magnétiques du sol et sur celles du schiste et du granite, Annales de Géophysique, 16, p. 159-195.

Le Borgne, E., 1965 - Les propriétés magnétiques du sol. Application à la prospection des sites archéologiques, Archaeophysika, 1, p. 1-21.

Leconnet H., Lévêque F. et Segura S., 1999 - Magnetic susceptibility in environmental applications: comparison of field probes. Phys. Earth Planet. Interiors, 115, p. 191-204.

Linford, N.T., 2004 -Magnetic Ghosts: Mineral magnetic measurements on Roman and Anglo-Saxon graves, Archaeological Prospection, 11, p. 167-180.
Marmet, E., Bina, M., Fedoroff, N. et Tabbagh, A., 1999 - A case study in the medieval site of Roissy-en-France, Archaeological Prospection, 6, p. 161-170.

Marmet, E., 2000 - Cartographie à large maille de la susceptibilité magnétique du sol pour une évaluation archéologique sur les grands tracés, thèse de doctorat, Université Pierre et Marie Curie, Paris 6, France.

Marshall, A., 2001 - Functional analysis of settlement areas: Prospection over a defended enclosure of Iron Age date at the Bowsings, Guiting Power, Gloucestershire, UK, Archaeological Prospection, 8, p. 79-106.

Mathé, V. et LÉvêQUe, F., 2003 - High resolution magnetic survey for soil monitoring: detection of drainage and soil tillage effects. Earth and Planetary Science,212,p . 241-251.

Mullins, C.E., 1974 - The magnetic properties of the soil and their application to archaeological prospecting, Archaeophysika, 5, p. 143-347.

Pétronille, M., Thiesson, J., Simon, F.-X. et Buschenschutz, O., 2010 - Magnetic signal prospecting using multiparameter measurements: the case study of the Gallic site of Levroux, Archaeological Prospection, 17, p. 141-151.

Schwartz, G.T., 1967 - A simplified chemical test for archaeological field work, Archaeometry, 10, p. 57-63.

Smith, H., Marshall, P. et Parker Pearson, M., 2001 Reconstructing houses activity areas, dans Albarella, U. (ed.). Environmental Archaeology: Meaning and Purpose. Environmental Science and Technology Library. Dordrecht/ Bolston/London, Kluwer, p. 249-270.

Tabbagh, J., 2003 - Total Field Magnetic Prospection: Are Vertical Gradiometer Measurements Preferable to Single Sensor Survey ?, Archeological Prospection, 10, p. 75-81.

Thiesson, J., 2007 - Mesure et cartographie de la viscosité magnétique des sols, thèse de doctorat, Université Pierre et Marie Curie, Paris 6, France.

Tite, M.S., Mullins, C.E., 1971 - Enhancement of the magnetic susceptibility of soils on archeological sites, Archaeometry, 13, p. 209-219.

Weston, D. G., 2002 - Soil and Susceptibility: Aspects of thermally induced magnetism within the dynamic pedological system, Archaeological Prospection, 9, p. 207-215. 